\title{
Positive epistasis between co-infecting plasmids promotes plasmid survival in bacterial populations
}

\author{
Alvaro San Millan, Karl Heilbron and R Craig MacLean \\ Department of Zoology, University of Oxford, Oxford, UK
}

\begin{abstract}
Plasmids have a key role in the horizontal transfer of genes among bacteria. Although plasmids are catalysts for bacterial evolution, it is challenging to understand how they can persist in bacterial populations over the long term because of the burden they impose on their hosts (the 'plasmid paradox'). This paradox is especially perplexing in the case of 'small' plasmids, which are unable to self-transfer by conjugation. Here, for the first time, we investigate how interactions between co-infecting plasmids influence plasmid persistence. Using an experimental model system based on interactions between a diverse assemblage of 'large' plasmids and a single small plasmid, pNI105, in the pathogenic bacterium Pseudomonas aeruginosa, we demonstrate that positive epistasis minimizes the cost associated with carrying multiple plasmids over the short term and increases the stability of the small plasmid over a longer time scale. In support of these experimental data, bioinformatic analysis showed that associations between small and large plasmids are more common than would be expected owing to chance alone across a range of families of bacteria; more generally, we find that co-infection with multiple plasmids is more common than would be expected owing to chance across a wide range of bacterial phyla. Collectively, these results suggest that positive epistasis promotes plasmid stability in bacterial populations. These findings pave the way for future mechanistic studies aimed at elucidating the molecular mechanisms of plasmid-plasmid interaction, and evolutionary studies aimed at understanding how the coevolution of plasmids drives the spread of plasmid-encoded traits.
\end{abstract}

The ISME Journal (2014) 8, 601-612; doi:10.1038/ismej.2013.182; published online 24 October 2013

Subject Category: Evolutionary genetics

Keywords: antibiotic resistance; epistasis; evolutionary genetics; experimental evolution; horizontal gene pool; plasmid

\section{Introduction}

Plasmids have a key role in bacterial adaptation to novel environments by providing bacterial populations with access to a pool of accessory genes that allow them to adapt to specific environmental stressors (Gogarten and Townsend, 2005; Smets and Barkay, 2005). For example, plasmids have had a pivotal role in the evolution of antibiotic resistance in pathogenic bacteria by allowing resistance genes to rapidly spread between bacterial strains (Alekshun and Levy, 2007; Martinez et al., 2009). In spite of the key role that plasmids have in bacterial evolvability, understanding how they can persist in bacterial populations in the absence of positive selection is challenging because of the following reasons: (i) plasmids generally impose a fitness burden on their hosts, producing a fitness advantage for plasmid-free segregants (Bouma and

Correspondence: A San Millan, Department of Zoology, University of Oxford, South Parks road, Oxford OX1 3PS, UK.

E-mail: alvaro.sanmillan@zoo.ox.ac.uk

Received 7 June 2013; revised 16 September 2013; accepted 17 September 2013; published online 24 October 2013
Lenski, 1988; Dahlberg and Chao, 2003; Enne et al., 2004; Dionisio et al., 2005; Yates et al., 2006; San Millan et al., 2010; Humphrey et al., 2012); (ii) sustained selection for plasmid-encoded traits can result in the movement of beneficial genes to the chromosome, rendering the plasmid redundant (Modi et al., 1992; Bergstrom et al., 2000; Toleman and Walsh, 2011); and (iii) about half of the plasmids are incapable of horizontal transmission by conjugation (Smillie et al., 2010). These asymmetries are thought to prevent the maintenance of plasmids as bacterial parasites in the absence of positive selection for plasmid-encoded genes (Gordon, 1992; Levin 1993). Hence, the long-term persistence of plasmids in bacterial populations is known as the 'plasmid paradox' (Harrison and Brockhurst, 2012). In this paper, we show that positive epistasis between co-infecting plasmids minimizes the cost of plasmid carriage and increases the ability of plasmids to persist in the absence of selection for plasmid-encoded traits, and we argue that epistasis may have an important role in resolving the 'plasmid paradox'.

Plasmids can be divided into two groups according to their size and biology: 'small' and 'large' 
(Smillie et al., 2010). Large plasmids may be capable of horizontally transferring themselves via conjugation, can potentially carry many beneficial genes and use active partitioning systems and postsegregational killing systems to prevent segregational loss of plasmids. Segregational loss would potentially occur at a relatively high frequency owing to the low copy number of large plasmids (typically $\approx 2$ ). In contrast, small plasmids lack conjugative machinery, carry few potentially beneficial genes and rely on a high copy number (typically $>10$ ) to avoid segregational loss. Therefore, it is especially challenging to understand how small plasmids persist in bacterial populations because they generate a burden that is similar to that produced by large plasmids (San Millan et al., 2010), but lack some of the mechanisms for stability within a host that large plasmids enjoy.

Our understanding of the evolution of bacteriaplasmid associations is largely based on the analysis of selection in systems containing a bacterial host and a single plasmid (Stewart and Levin, 1977; Freter et al., 1983; Simonsen, 1991; Bergstrom et al., 2000; Lili et al., 2007; Ponciano et al., 2007; Svara and Rankin, 2011; Harrison and Brockhurst, 2012). Co-infection of bacterial strains with multiple plasmids is common, but the influence of interactions between plasmids in evolution remains poorly understood (Paulsson, 2002). It is well known that the coexistence between plasmids that are members of the same incompatibility group is unstable because they share common elements involved in replication and partitioning (Novick, 1987). In this case, interactions between co-infecting plasmids compromise plasmid stability. However, it is also conceivable that interactions between compatible plasmids promote plasmid maintenance by either increasing the benefits associated with plasmid carriage or by decreasing its costs. These types of positive interactions that ameliorate bacterial fitness have been recently shown between chromosomal mutations and conjugative plasmids conferring antibiotic resistance (Silva et al., 2011).

In this work, we investigate the evolutionary consequences of interactions that occur between co-infecting 'large' and 'small' plasmids using an experimental system consisting of the bacterium $P$. aeruginosa and six plasmids that replicate in Pseudomonadaceae, including five large plasmids and a single small plasmid. We tested how interactions between large and small plasmids influence the costs (decreased competitive ability in the absence of antimicrobials) and the benefits (resistance to antibiotics) of plasmid carriage. To complement these short-term competition experiments, we carried out a long-term plasmid stability experiment in which we allowed populations of plasmidcarrying bacteria to evolve in the absence of antibiotics for 100 generations to determine how interactions between plasmids influence plasmid persistence in the absence of selection for plasmid- encoded genes. Finally, we analyzed data on the distribution of plasmids across all 1866 bacterial strains currently available in GenBank to test whether patterns of plasmid carriage in nature are consistent with the results of our experiments.

\section{Materials and methods}

A detailed version of the methods including the bioinformatic analysis is available as Supplementary Material.

Bacterial strains, plasmids and antibiotic susceptibility testing

The bacterial strains and plasmids used in this study are detailed in Table 1. Minimal inhibitory concentrations were performed in triplicate according to Clinical and Laboratory Standards Institute (2009).

\section{Competitive fitness assays}

The fitness of each strain carrying the different plasmid combinations was determined relative to the PAO1-green fluorescent protein (GFP) strain. Precultures of the strains were incubated at $37^{\circ} \mathrm{C}$ with 200 RPM shaking overnight in $3 \mathrm{ml}$ of LB broth (Fisher Scientific, Bridgewater, NJ, USA). Precultures were diluted 100-fold in $3 \mathrm{ml}$ of fresh LB broth and incubated in the same conditions until they reached mid-exponential phase $\left(\mathrm{OD}_{600}\right.$ of $\left.\approx 0.5\right)$. Cultures of the strains were then mixed at a ratio of $\approx 50 \% \mathrm{PAO} / \mathrm{plasmid}$ to $50 \% \mathrm{PAO} 1-\mathrm{GFP}$. The exact initial proportions were confirmed via flow cytometry using an Accuri C6 Flow Cytometer Instrument (BD Accuri, San Jose, CA, USA). Mixtures were diluted 200-fold in fresh LB and competed for $16 \mathrm{~h}$ at $37^{\circ} \mathrm{C}$ with no agitation ( $\approx 7$ generations). Again, the final proportion was measured by flow cytometry. The fitness of the strain carrying the plasmid(s) relative to the PAO1-GFP strain was determined using the formula $W_{\text {plasmid }}=\log _{e}\left(N_{\text {final,plasmid }} / N_{\text {initial, plasmid }}\right) / \log _{e}\left(N_{\text {final,GFP }} / N_{\text {initial,GFP }}\right)$, where $W_{\text {plasmid }}$ is the fitness of the strain carrying the plasmid, $\quad N_{\text {initial,plasmid }}$ and $N_{\text {final,plasmid }}$ are the numbers of cells of the strain carrying the plasmid before and after the competition and $N_{\text {initial,GFP }}$ and $N_{\text {final,GFP }}$ are the numbers of cells of PAO1-GFP before and after the competition.

\section{Quantification of plasmid copy numbers}

The copy numbers of plasmids were determined by quantitative polymerase chain reaction (qPCR) using an ABI Prism 7000 sequence detection system (Life Technologies, Paisley, UK). In Supplementary Table S2, we present the primers and conditions used for this analysis. To calculate the copy number of plasmid per chromosome, we used the formula: 
Table 1 Strains and plasmids used in this study

\begin{tabular}{|c|c|c|c|c|c|c|c|}
\hline \multirow{2}{*}{$\begin{array}{l}\text { Strain or plasmid } \\
\text { Plasmids }\end{array}$} & \multicolumn{6}{|c|}{ Description and characteristics } & \multirow[t]{2}{*}{ Reference, Accession no. ${ }^{\text {a }}$} \\
\hline & Group $^{\mathrm{b}}$ & $\begin{array}{l}\text { Size } \\
(k b)\end{array}$ & $\begin{array}{l}\text { Resistance } \\
\text { phenotype }^{\mathrm{c}}\end{array}$ & Transmission & Origin & Year & \\
\hline pNI105 & NA & 5 & $\mathrm{NeO}^{\mathrm{r}}, \mathrm{Kan}^{\mathrm{r}}$ & Non-transmissible ${ }^{\mathrm{d}}$ & Soil & 1991 & (Itoh et al., 1991) AB032347 \\
\hline pBS228 & IncP- $1 \alpha$ & 89 & Str $^{r}$, Carr $^{r}$, Tet $^{r}$ & Mobilizable & Waste water & 1981 & $\begin{array}{c}\text { (Haines et al., 2007) } \\
\text { NC_008357 }\end{array}$ \\
\hline Rms149 & IncP-6 & 57 & $\operatorname{Str}^{r}$, Gen $^{r}, \operatorname{Car}^{r}$ & Mobilizable & Clinical & 1975 & $\begin{array}{l}\text { (Haines et al., 2005) } \\
\text { NC_007100 }\end{array}$ \\
\hline pAKD1 & IncP-1 $\beta$ & 58 & $\mathrm{Str}^{\mathrm{r}}, \mathrm{Hg}^{\mathrm{r}}$ & Conjugative & Soil & 1998 & (Sen et al., 2011) JN106164 \\
\hline PAMBL-1 & ND & 30 & $\begin{array}{l}\operatorname{Gen}^{\mathrm{r}}, \operatorname{Car}^{\mathrm{r}}, \mathrm{Caz}^{\mathrm{r}} \\
\text { Merr }^{\mathrm{r}}, \mathrm{Hg}^{\mathrm{r}}\end{array}$ & Conjugative & Clinical & $2005-2007$ & $\begin{array}{l}\text { (Tato et al., 2010) } \\
\quad \text { GQ422829 }\end{array}$ \\
\hline PAMBL-2 & ND & 30 & $\begin{array}{l}\text { Strr }^{\mathrm{r}}, \text { Gen }^{\mathrm{r}}, \text { Carr }^{\mathrm{r}}, \\
\text { Caz }^{\mathrm{r}}, \mathrm{Mer}^{\mathrm{r}}\end{array}$ & Conjugative & Clinical & $2005-2007$ & $\begin{array}{l}\text { (Tato et al., 2010) } \\
\text { GQ422828 }\end{array}$ \\
\hline
\end{tabular}

Strains

\begin{tabular}{|c|c|}
\hline P. aeruginosa $\mathrm{PAO} 1$ & Susceptible strain used as a recipient for plasmids \\
\hline P. aeruginosa PAO1-GFP & $\begin{array}{l}\text { PAO1 tagged with the green fluorescent protein (GFP) } \\
\text { gene integrated at the Tn7 insertion site. } \mathrm{Gm}^{\mathrm{r}}, \mathrm{Ch}^{\mathrm{r}}\end{array}$ \\
\hline PAO1/ pNI105 & $\mathrm{Neo}^{\mathrm{r}}, \mathrm{Kan}^{\mathrm{r}}$ \\
\hline PAO1/ pBS228 & Strr $^{r}$, Carr $^{r}$, Tet $^{r}$ \\
\hline PAO1/ Rms149 & $\operatorname{Str}^{\mathrm{r}}, \mathrm{Gen}^{\mathrm{r}}, \mathrm{Car}^{\mathrm{r}}$ \\
\hline PAO1/ pAKD1 & Str ${ }^{r}, \mathrm{Hg}^{\mathrm{r}}$ \\
\hline PAO1/ PAMBL-1 & $\mathrm{Gen}^{\mathrm{r}}, \mathrm{Car}^{\mathrm{r}}, \mathrm{Caz}^{\mathrm{r}}, \mathrm{Mer}^{\mathrm{r}}, \mathrm{Hg}^{\mathrm{r}}$ \\
\hline PAO1/ PAMBL-2 & Str $^{\mathrm{r}}, \mathrm{Gen}^{\mathrm{r}}, \mathrm{Car}^{\mathrm{r}}, \mathrm{Caz}^{\mathrm{r}}, \mathrm{Mer}^{\mathrm{r}}$ \\
\hline PAO1/ pNI105 pBS228 & $\mathrm{Neo}^{\mathrm{r}}, \mathrm{Kan}^{\mathrm{r}}, \mathrm{Str}^{\mathrm{r}}, \mathrm{Car}^{\mathrm{r}}, \mathrm{Tet}^{\mathrm{r}}$ \\
\hline PAO1/ pNI105 Rms149 & $\mathrm{Neo}^{\mathrm{r}}, \mathrm{Kan}^{\mathrm{r}}, \mathrm{Gen}^{\mathrm{r}}, \mathrm{Str}^{\mathrm{r}}, \mathrm{Car}^{\mathrm{r}}$ \\
\hline PAO1/ pNI105 pAKD1 & $\mathrm{Neo}^{\mathrm{r}}, \mathrm{Kan}^{\mathrm{r}}, \mathrm{Str}^{\mathrm{r}}, \mathrm{Hg}^{\mathrm{r}}$ \\
\hline PAO1/ pNI105 PAMBL-1 & $\mathrm{Neo}^{\mathrm{r}}, \mathrm{Kan}^{\mathrm{r},} \mathrm{Gen}^{\mathrm{r}}, \mathrm{Car}^{\mathrm{r}}, \mathrm{Caz}^{\mathrm{r}}, \mathrm{Mer}^{\mathrm{r}}, \mathrm{Hg}^{\mathrm{r}}$ \\
\hline PAO1/ pNI105 PAMBL-2 & $\mathrm{Neo}^{\mathrm{r}}, \mathrm{Kan}^{\mathrm{r}}, \mathrm{Str}^{\mathrm{r}}, \mathrm{Gen}^{\mathrm{r}}, \mathrm{Car}^{\mathrm{r}}, \mathrm{Caz}^{\mathrm{r}}, \mathrm{Mer}^{\mathrm{r}}$ \\
\hline
\end{tabular}

NC_002516

(Choi and Schweizer, 2006)

This work

This work

This work

This work

This work

This work

This work

This work

This work

This work

This work

Abbreviations: NA, not applicable; ND, not determined.

aSequences present in GenBank for PAMBL-1, PAMBL-2 and pNI105 are partial.

${ }^{\mathrm{b} S}$ Small plasmid pNI105 does not have a defined group, it codes for a rolling circle replication-like rep-protein.

'Gen, gentamycin; Chl, chloramphenicol; Neo, neomycin; Kan, kanamycin; Str, streptomycin; Car, carbenicillin; Tet, tetracycline; Hg, mercury; Caz, ceftazidime; Mer, meropenem. ${ }^{\mathrm{r}}$, high-level resistance phenotype.

${ }^{\mathrm{d}}$ The small plasmid pNI105 showed a conjugation frequency $<10^{-7}$ transconjugants per donor colony-forming unit (no transconjugant detected in the conjugation assay) when coexisting with the conjugative plasmids pAKD1, PAMBL-1 and PAMBL-2.

cn $=\left(1+E_{\mathrm{C}}\right)^{\mathrm{Ctc}} /\left(1+E_{\mathrm{p}}\right)^{\mathrm{Ctp}} \times S_{\mathrm{c}} / S_{\mathrm{p}}$, where cn is the plasmid copy number per chromosome, $S_{\mathrm{c}}$ and $S_{\mathrm{p}}$ are the sizes of the chromosomal and plasmid amplicons (in bp), $E_{\mathrm{c}}$ and $E_{\mathrm{p}}$ are the efficiencies of the chromosomal and plasmid qPCRs (relative to 1) and Ctc and Ctp are the threshold cycles of the chromosomal and plasmid reactions, respectively.

\section{Results and discussion}

To investigate interactions between plasmids, we used six plasmids that are able to replicate in Pseudomonas and were recovered from different sources, including five large plasmids from a diverse range of incompatibility groups: pBS228, Rms149, pAKD1, PAMBL-1 and PAMBL-2, and a single small plasmid pNI105 (Itoh et al., 1991, 2003; Haines et al., 2005, 2007; Tato et al., 2010; Sen et al., 2011) (Table 1). The five large plasmids range in size from 30 to $90 \mathrm{~kb}$ and confer resistance to a wide variety of antibiotics and the heavy metal mercury (Table 1). Three of these large plasmids are conjugative (pAKD1, PAMBL-1 and PAMBL-2) and two are mobilizable (pBS228 and Rms149, that is, they are not self-transmissible but they can be transferred by a trans-acting conjugative element present in the same host bacteria). The small plasmid pNI105 is only $5 \mathrm{~kb}$ in length and confers resistance to neomycin and kanamycin; this plasmid cannot be mobilized by any of the large plasmids used in this study under our experimental conditions (see Materials and methods). To study interactions between large and small plasmids, $P$. aeruginosa PAO1 was transformed with the six different plasmids independently and with all five smalllarge plasmid combinations (Table 1).

\section{Positive epistasis minimizes the burden of plasmid carriage}

To determine how epistasis between plasmids influences the burden associated with plasmid carriage, we directly competed plasmidbearing strains against a plasmid-free strain (PAO1) in culture medium lacking antibiotics. We distinguished between plasmid-bearing and plasmid-free cells by using a plasmid-free strain 
carrying a GFP gene integrated at the Tn7 insertion site (Choi and Schweizer, 2006). As a control for the costs and benefits associated with this construct, we also competed the GFP-tagged strain (PAO1-GFP) against an unmarked PAO1 strain (the average fitness of PAO1-GFP relative to PAO1 was 0.977, s.e. $=0.005$; $n=5$ ). There was significant variation in the cost of carriage among large plasmids (one-way analysis of variance: $P=0.0002, \mathrm{~F}=17.70, \mathrm{df}=4,10$ ), with some plasmids lacking a detectable cost and others imposing a substantial burden of up to $14.3 \%$ (Figure 1a). Consistent with previous work, we found that the small plasmid imposed a large cost (mean fitness cost $=13.2 \%$; s.e. $=2.5 \% ; n=3$ biological replicates of 5 technical replicates each).

Epistasis between plasmids (e) was calculated as previously described by Silva et al., 2011, using the formula $\mathrm{e}=W_{\text {S.L }}-W_{\text {S- }} \times W_{\text {-L }}$, where $W_{\text {S- }}, W_{\text {-L }}$ and $W_{\text {S.L }}$ are the fitnesses (relative to the plasmid-free PAO1) of the strains carrying a small plasmid, a large plasmid and both small and large plasmids, respectively, and the error of $e\left(\sigma_{\mathrm{e}}\right)$ was estimated by the method of error propagation (Trindade et al., 2009; Hall and MacLean, 2011; Silva et al., 2011). As in Silva et al. (2011), we interpreted a positive value for $e-\sigma_{\mathrm{e}}$ as significant positive epistasis between plasmids. On the whole, we found evidence that strong positive epistasis minimizes the burden associated with carrying multiple plasmids. We detected significant positive epistasis in $4 / 5$ plasmid associations (pNI105 and Rms149 $e=0.119 \pm 0.065$, pBS228 $e=0.125 \pm 0.05$, pAKD1 $e=0.159 \pm 0.068$, PAMBL-2 $e=0.085 \pm 0.076$ ) and in the remaining case the interaction between pNI105 and PAMBL-1 did not differ significantly from the multiplicative model $(e=0.005 \pm 0.068)$. In the four associations showing positive interactions, strains carrying a large plasmid paid very low costs or no additional costs at all for acquiring the small plasmid, in spite of the large cost associated with introducing a small plasmid into a plasmid-free strain (Figure 1b).

\section{Co-infection does not reduce plasmid copy number}

Previous work has shown that the burden associated with plasmid carriage correlates to plasmid copy number (Patnaik, 2000; Harrison et al., 2012). To test the hypothesis that a reduction in plasmid copy number drives positive epistasis between plasmids, we measured the number of copies of each plasmid per cell using qPCR (Figure 2). As expected, the copy number of the small plasmid (18 copies/cell; s.e. $=2.4$ ) was substantially higher than the copy number of the large plasmids, which ranges between $\approx 1$ and 3 . Across all of the plasmid-plasmid associations, we found that acquiring a large plasmid did not significantly change the copy number of the small plasmid (two-sample $t$-tests: false discovery rate-corrected $P>0.4$ for all combinations, see the legend of Figure 2; Benjamini and Hochberg, 1995). Similarly, acquiring a small plasmid did not significantly change the average copy number of any of the large plasmids (Two-sample $t$ tests: false discovery rate-corrected $P>0.1$ for all combinations, see the legend of Figure 2; Benjamini and Hochberg, 1995). In summary, the coexistence of multiple plasmids in the same cell did not lead to any consistent changes in plasmid copy number, demonstrating that reduced plasmid copy number cannot explain the low cost associated with carrying multiple plasmids.

\section{Plasmids contribute independently to antibiotic} resistance

Recent studies have shown that the cross talk between large plasmids and the bacterial chromo-
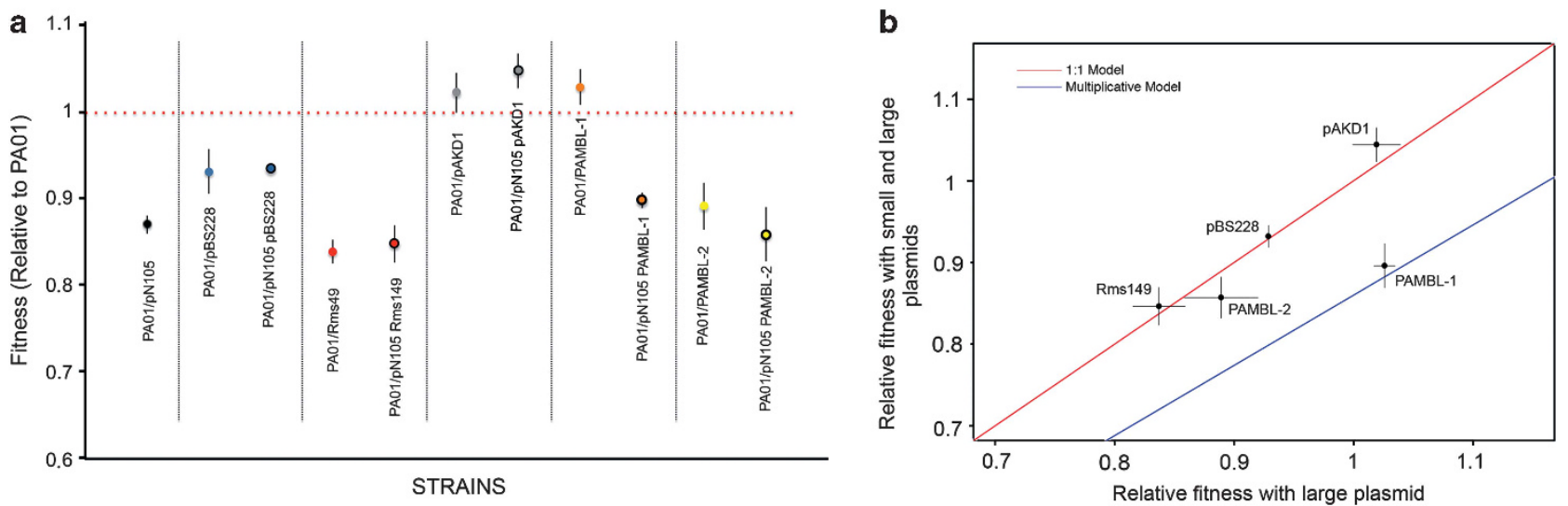

Figure 1 Fitness of plasmid-carrying strains. (a) Fitness ( \pm s.e.) of PAO1 carrying one and two plasmids, relative to the plasmid-free ancestor, P. aeruginosa PAO1. The horizontal dotted red line represents the fitness of PAO1. Vertical dotted black lines separate each pair of strains carrying a large plasmid alone and combined with the small plasmid pNI105. (b) Positive epistasis between large and small plasmids. Comparison of the relative fitness of $P$. aeruginosa PAO1 carrying large and both large and small plasmids. The blue line represents the expected fitness if the fitness cost produced by large and small plasmids is multiplicative. The red line represents the expected fitness if the small plasmid (pNI105) produces no extra cost. Error bars represent standard error. The points above the blue line represent associations of plasmids showing positive epistasis. 


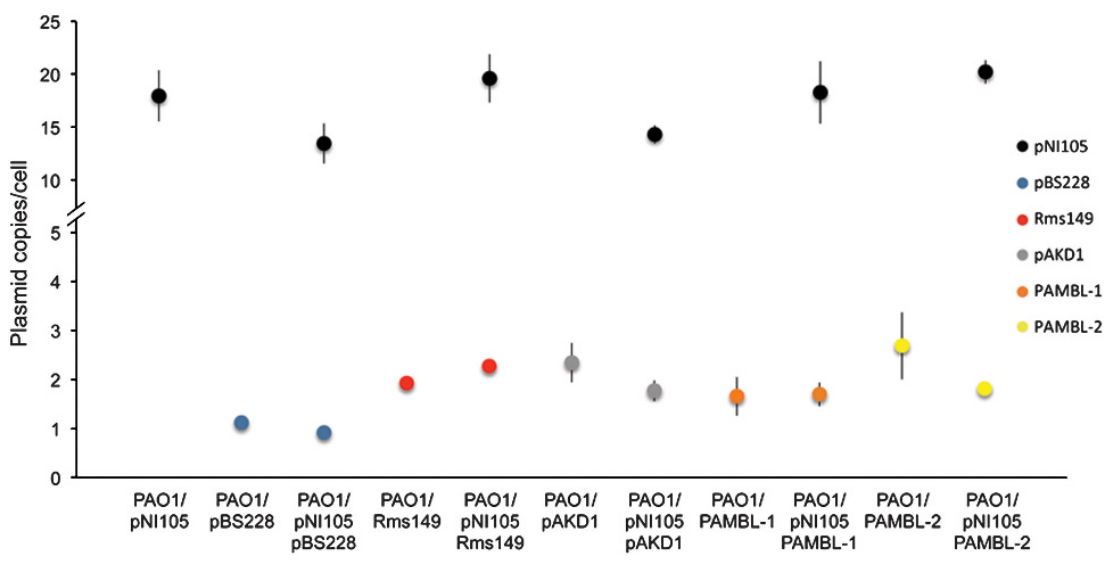

Figure 2 Plasmid copy numbers in PAO1-transformed strains. Average number of plasmid copies per cell ( \pm s.e.). Copy numbers were determined by qPCR of plasmid-encoded genes using the chromosomal gene $r p o D$ as a reporter. The copy number of the small plasmid is not altered by the coexistence with a large plasmid (two-sample $t$-tests: pNI105 and Rms149: $P=0.785$; pNI105 and pBS228: $P=0.454$; pNI105 and pAKD1: $P=0.454$; PAMBL-1: $P=0.935$; PAMBL-2: $P=0.684 ; \mathrm{df}=10$ ). The presence of the small plasmid does not change the copy number of large plasmids (two-sample $t$-tests: Rms149: $P=0.104$; pBS228: $P=0.104$; pAKD1: $P=0.271$; PAMBL-1: $P=0.932$; PAMBL-2: $P=0.272 ; \mathrm{df}=4)$.

some produces alterations in gene expression that minimize the fitness cost associated with plasmid carriage (Harr and Schlötterer, 2006; Navarre et al., 2006; Doyle et al., 2007; Shintani et al., 2010; Yun et al., 2010). An alternative explanation for the reduction in the cost of coexisting plasmids that we observed could be that plasmid gene expression decreases during co-infection. To test this hypothesis, we measured the resistance of strains carrying single plasmids or pairs of plasmids to antibiotics and the heavy metal mercury, as defined by their minimal inhibitory concentration (Supplementary Figure S1; Supplementary Table S1). All of the plasmids in this study carry antibiotic resistance genes (Table 1) that degrade or inactivate antibiotics, and it is well established that phenotypic levels of resistance for this class of resistance genes are well correlated with enzyme expression levels (Uhlin and Nordström, 1977; Mick et al., 2008). We found that coexisting plasmids contributed additively to antibiotic resistance, as demonstrated by the fact that acquiring a plasmid that did not confer resistance to a particular antibiotic did not change the level of resistance (that is, minimal inhibitory concentration) of cells harboring a plasmid conferring resistance to that antibiotic (paired $t$-test: $P=0.752, \quad t=-0.318, \quad \mathrm{df}=44, \quad$ Supplementary Figure S1). When both plasmids conferred resistance (pNI105 confers high-level resistance to kanamycin and neomycin, and PAMBL-1 and PAMBL-2 confer very low-level resistance to kanamycin and to kanamycin and neomycin, respectively), the strains carrying both of them showed an additive effect on their resistance level (Supplementary Table S1, Supplementary Figure S1). These results provide good evidence that positive epistasis between plasmids does not arise as a consequence of the repression of plasmidencoded antibiotic resistance genes. In the broader context of antibiotic resistance, this implies that acquiring multiple plasmids provides bacteria with a mechanism to become resistant to multiple antibiotics without incurring a large fitness cost. Coexistence of antibiotic resistance plasmids producing positive epistasis might therefore have a key role in the maintenance of multidrug resistance phenotypes in nature. These results could help explain why multidrug resistance in clinical pathogens is sometimes associated with carrying multiple resistance plasmids (San Millan et al., 2009; Granier et al., 2011; Wei et al., 2011).

\section{Positive epistasis favors plasmid stability}

As plasmids impose substantial fitness costs, it is challenging to explain how plasmids can be stably maintained over long time scales. In theory, positive epistasis between plasmids could help maintain the stability of plasmids in the absence of selection for plasmid-encoded genes by minimizing the benefits associated with losing individual plasmids. Specifically, under positive epistasis, co-infecting plasmids mask each other's costs so that the loss of either plasmid individually will generate only a marginal fitness benefit to the bacterium. Conversely, if plasmids do not interact (multiplicative effects on fitness), plasmid stability would not be increased because the benefit associated with losing a plasmid is the same irrespective of whether or not the plasmid co-occurs with a second plasmid (Figure 3).

To test the hypothesis that plasmid coexistence increases stability, we analyzed the stability of the plasmids both alone and in combination. We allowed strains of bacteria carrying pNI105 either individually or in combination with one large plasmid to propagate in an antibiotic-free culture medium. After 100 generations of growth in the 


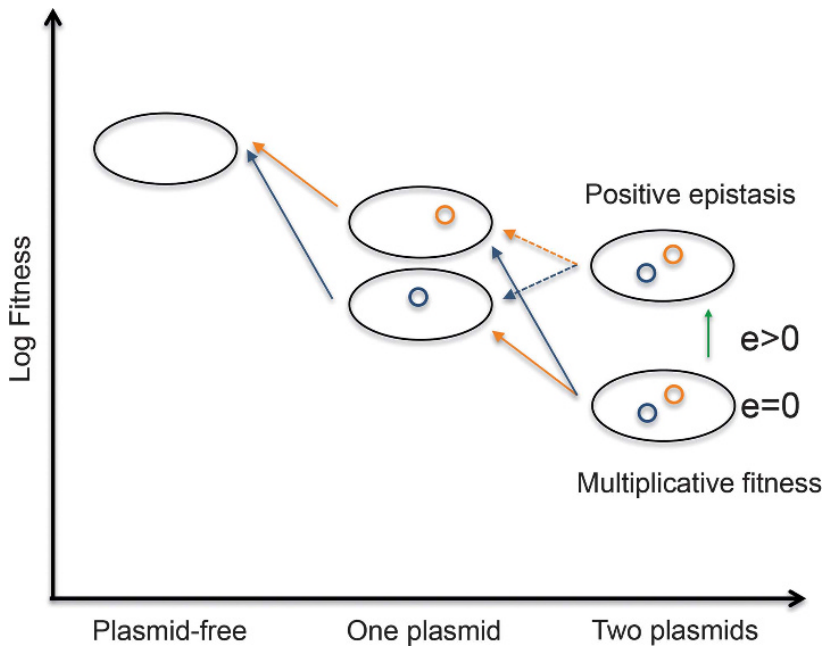

Figure 3 Positive epistasis increases plasmid stability. A schematic representation of the fitness of a bacterial strain carrying zero, one or two plasmids. In this example, the strain carrying two plasmids can present either multiplicative fitness (no epistasis between plasmids, $e=0$ ) or positive epistasis between plasmids $(e>0)$. The arrows represent the change in fitness associated with the loss of a plasmid. The strain carrying two plasmids that produce multiplicative costs $(e=0)$ will experience the same fitness advantage associated with the loss of each of the plasmids as in the strain that carries the single plasmids (orange and blue arrows, the increase in fitness is the same). On the other hand, in the case of positive epistasis, the advantage associated with the loss of a plasmid will be lower than in the previous cases (orange dotted arrow); the loss of the plasmid would even produce a decrease in fitness if the fitness of the strain with two plasmids is higher than the fitness of the strain with only one of the plasmids (blue dotted arrow).

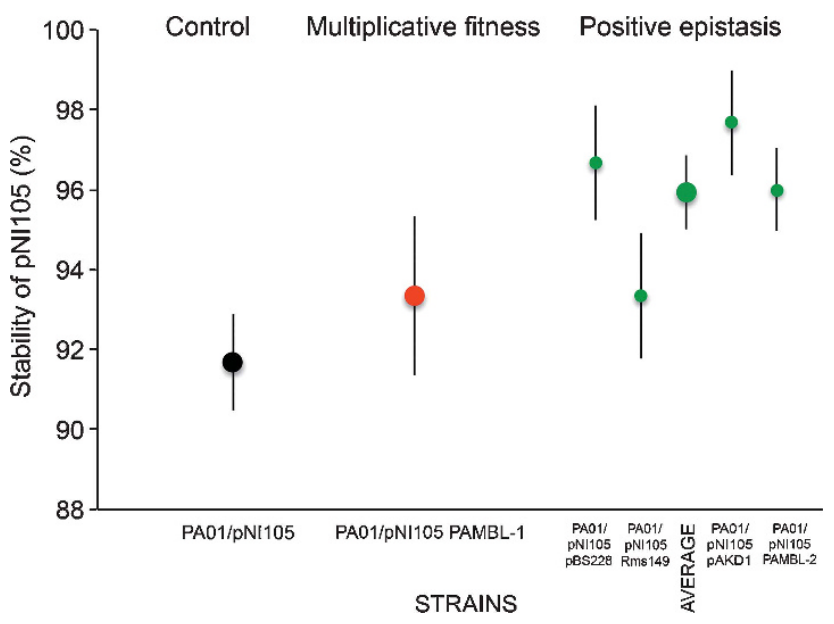

Figure 4 Stability of the small plasmid, pNI105, after evolution. Stability of pNI105 (percentage of plasmid-bearing cells, \pm s.e.) in the populations with the different plasmid combinations after 100 generations of evolution. PAO1/pNI105 is the control strain, carrying pNI105 alone. The plasmids of the PAO1/pNI105 PAMBL-1 strain display multiplicative fitness effects. The remaining plasmid combinations display positive epistasis and are grouped together. In the four populations where pNI105 is both associated with a large plasmid and also displays positive epistasis, there are no significant differences in the stability of pNI05 among them (one-way analysis of variance, $P=0.193$, $\mathrm{F}=1.73, \mathrm{df}=3,20)$. 'Average' is the average of the results ( \pm s.e.) of the stability of pNI105 in the strains showing positive epistasis between plasmids. absence of antibiotics, we measured the percentage of plasmid-bearing cells in each population (for every plasmid) as a measure of plasmid stability (Figure 4). Large plasmid stability was high. Rms149, pAKD1, PAMBL-1 and PAMBL-2 were present in $\geqslant 99 \%$ of the colonies tested both when they were alone and in combination with pNI105. pBS228 showed a lower stability $(80.3 \%$ alone and $87 \%$ in combination with pNI105) probably owing to the particularly low copy number per cell of this plasmid in PAO1 (Figure 2). The stability of the small plasmid was high, but variable, and, as expected, we found differences in the stability of pNI105 across treatments (analysis of variance: $P=0.0185, \mathrm{~F}=3.645, \mathrm{df}=2,33)$. The stability of pNI105 was lowest (mean $=91.7 \%$; s.e. $=1.2 \% ; n=6$ ) in the control treatment that only carried pNI105. In the four strains carrying combinations of plasmids that displayed positive epistasis, the average stability of pNI105 (95.9\%; s.e. $=0.93 \%$; $n=4)$ was significantly higher than in the pNI105alone control strain (Dunnett's test: $P=0.016$, $t=2.493, \mathrm{df}=2,33$ ), consistent with the hypothesis that positive epistasis between plasmids increases plasmid stability. Further evidence to support this hypothesis comes from the observation that the stability of pNI105 when coexisting with PAMBL-1, a plasmid that showed almost perfect multiplicative fitness with pNI105 (mean stability $=93.3 \%$; s.e. $=1.98 \%$ ), is not significantly different from the stability in the pNI105-alone control strain (Dunnett's test: $P=0.325, t=0.773, \mathrm{df}=2,33$ ).

In general, the results of the stability experiments showed an overall tendency for higher stability for plasmid pNI105 when coexisting with large plasmids with which it exhibits positive epistasis. Taking into account that pNI105 is not mobilizable in this model system, these results support the hypothesis that positive epistasis may have a role in increasing plasmid persistence in bacterial populations.

Small and large plasmids are associated in nature One of the limitations of this work is that we only had access to one small plasmid conferring resistance to antibiotics for the PAO1 model. Therefore, we decided to extrapolate beyond our experimental system using a bioinformatic approach to test for signatures of increased plasmid stability stemming from positive epistasis between large and small plasmids. We analyzed the patterns of distribution of plasmids across the genomes of free-living bacteria available in GenBank. If positive epistasis between large and small plasmids has a key role in the maintenance of small plasmids, then we expect the following: (a) strains carrying only small plasmids should be under-represented, (b) strains carrying a combination of large and small plasmids should be over-represented and/or they should carry a disproportionately high frequency of small plasmids.

To analyze the distribution of large and small plasmids, it was necessary to establish a size limit 
between those two groups. In the complete genome database (1866 genomes, see methods), this limit is not evident (Supplementary Figure S2) owing to the heterogeneity in plasmid biology and sizes among the different bacterial taxa. Specifically, there is a positive correlation between chromosome and plasmid lengths (Smillie et al., 2010) that masks the specific distribution of plasmid size within each group when all the groups are pooled. Therefore, we analyzed the distribution of size within bacterial families where plasmid biology and genome size are more homogeneous. Those families having $\geqslant 20$ genomes carrying plasmids were further analyzed: Enterobacteriaceae (174 genomes), Bacillaceae (76 genomes), Staphylococcaceae (42 genomes) and Lactobacillaceae (43 genomes). In three of these families (Enterobacteriaceae, Bacillaceae and Staphylococcaceae), the distribution of plasmid by size is bimodal and a clear limit could be established to perform the analysis (Figure 5; for the distribution in the Lactobacillaceae family, see Supplementary Figure S3). The plasmid-bearing genomes of these three families were analyzed. Three categories of strains were designated: strains with small plasmids (one or more, no large plasmids), strains with large plasmids (one or more, no small plasmids) and strains with both large and small plasmids (one or more of each). The expected frequencies of strains in each category, as well as the small and large plasmid frequencies within each category, were calculated for each family under the null hypothesis that associations between large and small plasmids are random. We then tested our two hypotheses and found a deficit in strains carrying only small plasmids both in Enterobacteriaceae $\left(\chi^{2}\right.$ test, $\left.P<0.0001, \chi^{2}=15.80, \mathrm{df}=1\right)$ and Staphylococcaceae $\left(\chi^{2}\right.$ test, $\left.P=0.034, \chi^{2}=4.51, \mathrm{df}=1\right)$, but not in Bacillaceae ( $\chi^{2}$ test, $P=0.523, \chi^{2}=0.41, \mathrm{df}=1$ ) (Figure 5b). The number of strains carrying both large and small plasmids was not significantly increased in any of the families $\left(\chi^{2}\right.$ test: Enterobacteriaceae, $P=0.501, \chi^{2}=0.45, \mathrm{df}=1$; Bacillaceae, $P=0.709, \quad \chi^{2}=0.14, \quad \mathrm{df}=1 ; \quad$ Staphylococcaceae, $\left.P=0.95, \chi^{2}=0.01, \mathrm{df}=1\right)$. On the other hand, the frequency of small plasmids in the strains carrying both large and small plasmids was higher than expected by chance for both Enterobacteriaceae $\left(\chi^{2}\right.$ test, $\left.P<0.0001, \chi^{2}=41.67, \mathrm{df}=1\right)$ and Bacillaceae $\left(\chi^{2}\right.$ test, $\left.P=0.02, \chi^{2}=5.35, \mathrm{df}=1\right)$, but not for Staphylococcaceae $\left(\chi^{2}\right.$ test, $P=0.25, \quad \chi^{2}=1.34$, $\mathrm{df}=1$ ) (Figure 5c). Therefore, at least one of the two predictions supporting the tendency for
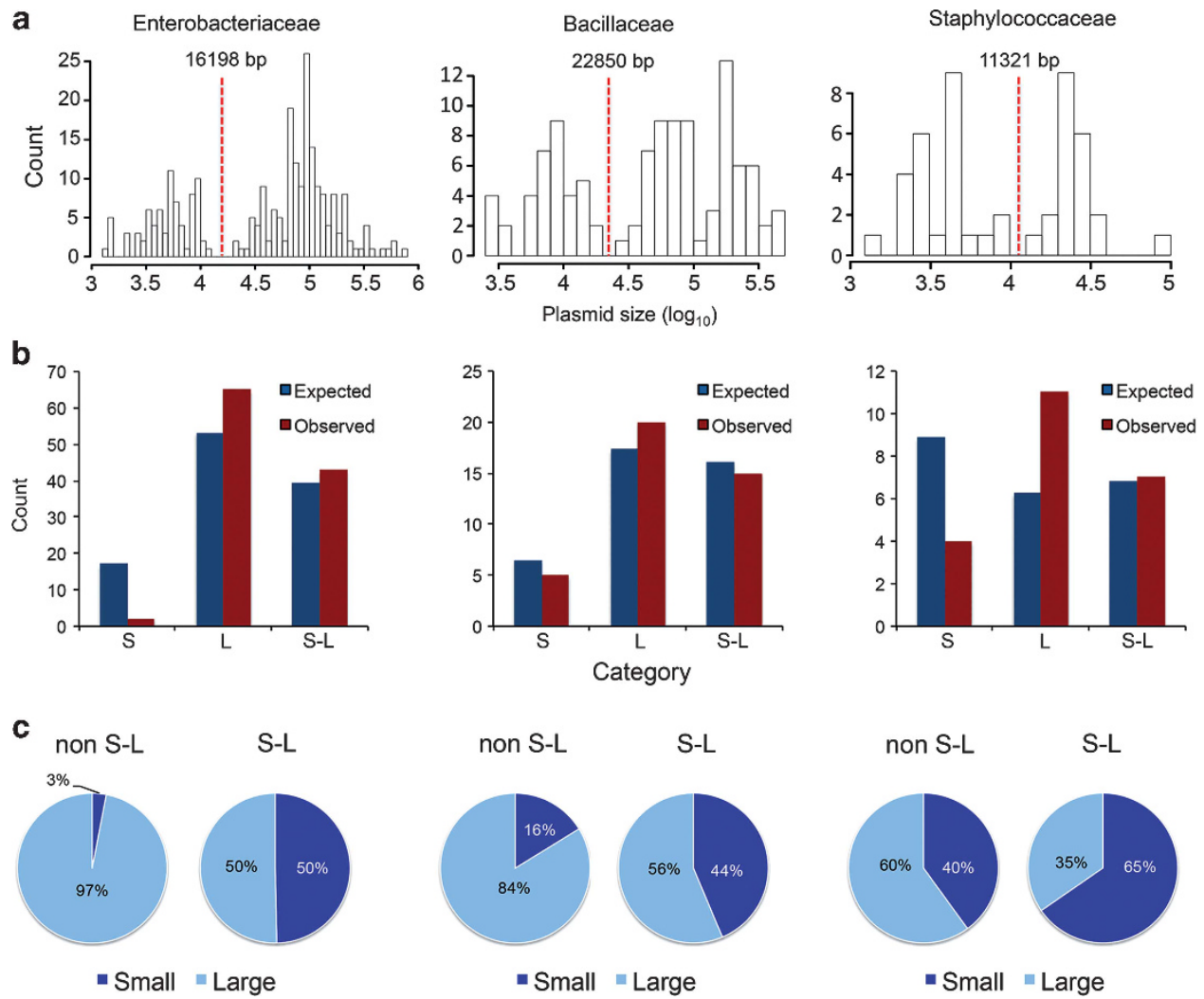

Figure 5 Distribution of small and large plasmids in Enterobacteriaceae, Staphylococcaceae and Bacillaceae. (a) Bimodal distribution of plasmid size in different bacterial families. The dashed red line represents the threshold size between small and large plasmids in each family. (b) Analysis of the distribution of small and large plasmids. The blue bars represent the expected number of genomes carrying one or more small plasmids (S, no large plasmids), one or more large plasmids (L, no small plasmids) or both small and large plasmids (S-L, one or more of each) under the null assumption of no association between plasmid size classes. The red bars represent the actual number of genomes in each category. (c) The percentage of small (dark blue) and large plasmids (light blue) in the strains carrying both small and large plasmids at the same time (S-L) and in the rest of plasmid-carrying genomes (non S-L) of the different families. 
small-large plasmid associations was confirmed in each of the three different families tested. Collectively, these bioinformatic results are consistent with the hypothesis that positive epistasis between large and small plasmids is common, and that interactions with large plasmids could have a key role in stabilizing small plasmids. This analysis reveals that in three of the most clinically relevant bacterial families there is a tendency for associations between small and large plasmids, especially in Enterobacteriaceae. Interestingly, these families present numerous examples of small plasmids carrying antibiotic resistance genes (Bernhard et al., 1978; Cao et al., 2002; Bjorland et al., 2003; Hauschild et al., 2006; Zioga et al., 2009; Chen et al., 2010; Kadlec et al., 2010; Wang et al., 2012), and thus these associations could lead to the acquisition of multiresistance phenotypes, which represent a serious concern for infectious disease therapeutics.

Alternatively, it could be argued that this tendency for associations between large and small plasmids arises from the fact that mobilizable small plasmids cannot transmit horizontally unless they co-occur with a large plasmid. Previous analysis based on the presence of mobilization (relaxases) genes showed that approximately half of the small plasmids are supposed to be non-mobilizable (Smillie et al., 2010), and thus they would not be able to co-transfer with large plasmids. Using the same approach, we found that exactly $50 \%$ (70) of the small plasmids in these three bacterial families are non-mobilizable, and the distribution of non-mobilizable small plasmids does not differ significantly $\left(\chi^{2}\right.$ test, $P=0.783, \chi^{2}=0.08$, $\mathrm{df}=1$ ) between genomes with only small plasmids (8 out of 17) and genomes with both large and small plasmids (62 out of 123). This analysis suggests that the tendency for the coexistence of small and large plasmids is, at minimum, not solely driven by increased horizontal transmission of small plasmids in the presence of large plasmids. The caveat to this analysis is that defining the potential for plasmid mobilization based on relaxases genes may underestimate the mobilizable capacity of small plasmids as they could be using trans-acting or as-yet unknown relaxases.

\section{Co-infection with multiple plasmids is common}

Although our experimental work focused on studying interactions between one small and five large plasmids, it is conceivable that positive interactions shape the evolution of compatible plasmid coexistence regardless of size. If positive epistasis between plasmids is a general phenomenon, then we expect that the frequency of strains carrying a single plasmid will be under-represented relative to strains carrying multiple plasmids.

To test this prediction, we analyzed the number of plasmids present in the 1866 bacterial genomes that are currently available in GenBank. Our null hypothesis is that if plasmids are distributed randomly across bacteria, then the number of plasmids per genome will be Poisson distributed. Our justification for this null hypothesis is that if $n$ observations (in this case, plasmids) are randomly distributed across $x$ discrete intervals (in this case, genomes), then the distribution of observations per interval (in this case, plasmids/genome) will follow the Poisson distribution (Zar, 1974). The Poisson distribution provides a useful statistical null model, because a significant deviation from the Poisson distribution implies that chance alone cannot explain the observed distribution of plasmids across bacterial genomes and that other forces, such as selection and conjugation, must influence this distribution. We found that the distribution of plasmids per strain deviated significantly from the Poisson expectation (Figure 6a, $\chi^{2}$ test, $P<0.0001$, $\chi^{2}=1045.31, \mathrm{df}=4$ ), which is perhaps not surprising because selection will influence the distribution of plasmids. For example, we found that strains that lack plasmids are more common than we would expect owing to chance $\left(\chi^{2}\right.$ test, $P<0.0001$, $\left.\chi^{2}=271.43, \mathrm{df}=1\right)$, and strains carrying one plasmid are less common than expected ( $\chi^{2}$ test, $P<0.0001$, $\chi^{2}=305.31, \mathrm{df}=1$ ), which is consistent with selection acting against strains bearing plasmids owing to the cost of plasmid carriage. Given that selection against plasmids is effective, strains carrying multiple plasmids should become progressively more under-represented as plasmid number increases if fitness interactions between plasmids are multiplicative or negative. In contrast to this idea, we found that strains carrying multiple $(>2)$ plasmids are over-represented ( $\chi^{2}$ test, $P<0.0001, \chi^{2}=136.66$, $\mathrm{df}=1$ ); specifically, we found that the overrepresentation of strains carrying multiple plasmids increases exponentially with plasmid number, as we would expect if positive epistasis occurs between plasmids (analysis of variance: $P=0.0003$, $\mathrm{F}=78.784, \quad \mathrm{df}=1, \quad 5, \quad \mathrm{R}^{2}=0.928$ ) (Figure 6b). Although these findings are consistent with positive epistasis facilitating multiple plasmid coexistence, it is important to keep in mind that the majority of plasmid-positive strains still carry only one or two plasmids (454 out of 629 ).

It could be argued that the tendency for plasmids to coexist in these genomes is a consequence of the bias toward sequencing strains of clinical origin, where antibiotics impose a strong pressure and preferentially select bacteria carrying coexisting antibiotic resistance plasmids. To address this possibility, we performed the same plasmid distribution analysis for all bacterial phyla with $\geqslant 30$ genomes sequenced and $\geqslant 10$ genomes carrying plasmids: Proteobacteria (819 genomes), Bacteroidetes (72 genomes), Actinobacteria (229 genomes), Cyanobacteria (48 genomes) and Firmicutes (475 genomes). These phyla differ in the proportion of clinically relevant species that they contain; in fact, the phylum Cyanobacteria includes no species of clinical significance at all. Regardless of the 


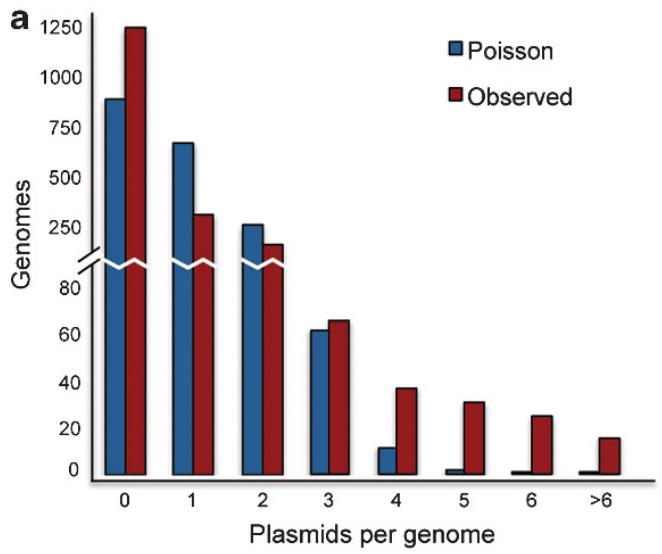

b

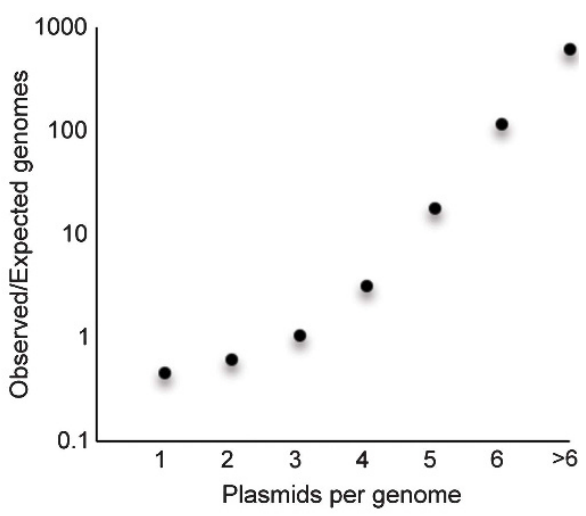

Bacteria

Proteobacteria

Bacteroidetes
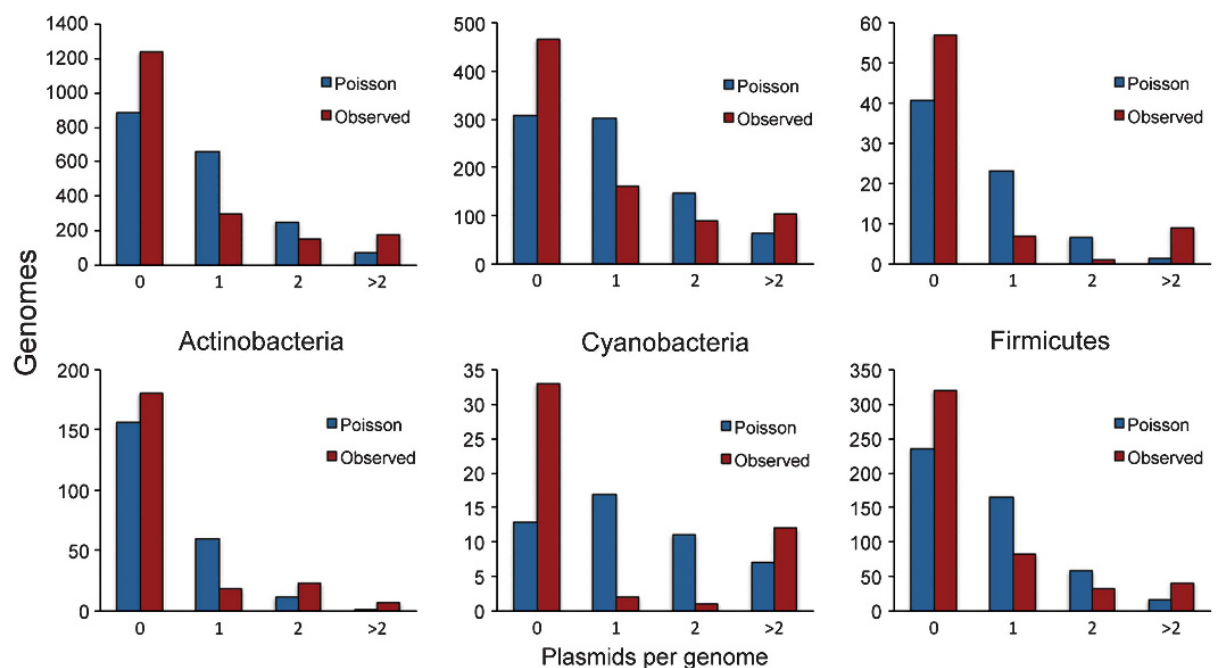

Figure 6 Distribution of plasmids in bacterial genomes. (a) Number of plasmids per bacterial genome. The blue bars represent the expected number of genomes carrying from zero to $>6$ plasmids following a Poisson distribution (using the average plasmid/strain observed in the 1866 bacterial genomes analyzed from GenBank). The red bars represent the observed frequency of strains carrying zero to $>6$ plasmids in the bacterial genomes analyzed. (b) Distribution of plasmid-bearing strains, relative to expected. The ratio of observed over expected (under the Poisson distribution) number of strains bearing various numbers of plasmids. There is an exponential increase in the observed/expected ratio as the number of plasmids per strain increases ( $y$-axis is presented with a logarithmic scale). Observed/expected $=0.0504 e^{1.2475 \times \text { number of plasmids }}$. (c) Plasmid distribution in all bacterial phyla with $\geqslant 30$ genomes sequenced and $\geqslant 10$ genomes carrying plasmids. The blue bars represent the expected number of genomes carrying from zero to $>2$ plasmids following a Poisson distribution (using the average plasmid/strain observed in each group). The top left-hand panel represents the distribution for all the bacterial genomes pooled together (Bacteria). The remaining panels represent the distribution in each different phylum analyzed (Proteobacteria, Bacteroidetes, Actinobacteria, Cyanobacteria and Firmicutes).

representation of clinically relevant species, the same main features were observed in every group when compared with what was predicted by the Poisson distribution (Figure 6c): (i) a nonrandom distribution of plasmids ( $\chi^{2}$ test, $P<0.0005$ ), (ii) a higher frequency of strains with zero plasmids $\left(\chi^{2}\right.$ test, $P<0.001, \mathrm{df}=1$ ), (iii) a lower frequency of strains with one plasmid ( $\chi^{2}$ test, $P<0.0001, \mathrm{df}=1$ ) and (iv) a higher frequency of genomes with $>2$ plasmids ( $\chi^{2}$ test, $P<0.05$, df $=1$ ). In Actinobacteria and Bacteroidetes, this last analysis could not be performed because the expected frequency of strains with $>2$ plasmids was lower than five, but the observed number was considerably larger than expected in both cases (Actinobacteria: expected $=1.56$, observed $=7 . \quad$ Bacteroidetes: $\quad$ expected $=1.45$, observed =9). Finally, in four out of the five phyla (Proteobacteria, Bacteroidetes, Cyanobacteria and Firmicutes), strains carrying two plasmids were more rare than expected ( $\chi^{2}$ test, $P<0.02, \mathrm{df}=1$ ), whereas in Actinobacteria they were more frequent than expected ( $\chi^{2}$ test, $\left.P<0.001, \mathrm{df}=1\right)$. Notably, in all the different phyla, the same general tendency toward plasmid coexistence was observed (regardless of plasmid size), indicating that this is a general phenomenon occurring across the bacteria phylogeny and independent of the antibiotic pressure experienced by bacterial pathogens. Interestingly, the distribution of plasmids in bacterial hosts that we observed is similar to the classic negative binomial distribution observed for metazoan parasites infecting individual hosts in a population 
(Shaw and Dobson, 1995; Poulin, 2013). Other biases may be present in the databases, such as the loss of plasmids during genome assembly, and thus even when we consider that the bioinformatic analyses support the hypothesis that positive epistasis has a role in plasmid stability in bacterial populations these results should be interpreted with caution.

\section{Conclusion}

It is important to understand the underlying evolutionary biology of bacteria-plasmid associations because of the key role that plasmids have in bacterial evolution. The central unresolved problem in our comprehension of bacteria-plasmid interactions is to understand how plasmids can persist in bacterial populations in the absence of selection for plasmid-encoded genes. Previous models and experiments have focused on tackling this problem in systems containing a bacterium and a single plasmid (Stewart and Levin, 1977; Freter et al., 1983; Simonsen 1991; Levin 1993; Verger et al., 1993; Bergstrom et al., 2000; Lili et al., 2007; Ponciano et al., 2007); ironically, we find that strains carrying a single plasmid are relatively rare (Figure 6), and that the coexistence of multiple plasmids in the same strain is relatively common (Figure 6). Our experimental work in $P$. aeruginosa shows that positive epistasis between plasmids increases plasmid stability, and bioinformatic analysis makes the tantalizing suggestion that positive epistasis may have a key important role in resolving the 'plasmid paradox' across bacteria. We hope that this study will pave the way for future mechanistic studies aimed at understanding why positive epistasis occurs between plasmids, and evolutionary studies aimed at understanding how positive epistasis impacts the coevolution of bacteria and plasmids.

\section{Conflict of Interest}

The authors declare no conflict of interest.

\section{Acknowledgements}

We thank Nobuya Itoh, Christopher M Thomas, Eva M Top and Maria Teresa Coque for kindly providing the plasmids for this study. The research leading to these results has received funding from the European Research Council under the European Union's Seventh Framework Programme (FP7/2007-2013)/ERC grant agreement no. 281591 and from the Royal Society. The Ministerio de Educación, Cultura y Deporte of Spain and a Marie Curie Intra-European Fellowship within the 7th European Community Framework Programme (PIEF-GA-2011-298975) supported the work of ASM. KH was supported by funding from the Natural Sciences and Engineering Research Council of Canada (NSERC), the Clarendon Fund and The Queen's College, University of Oxford.

\section{References}

Alekshun MN, Levy SB. (2007). Molecular mechanisms of antibacterial multidrug resistance. Cell 128: 1037-1050.

Benjamini Y, Hochberg Y. (1995). Controlling the false discovery rate: a practical and powerful approach to multiple testing. J $R$ Stat Soc B 45: 289-300.

Bergstrom CT, Lipsitch M, Levin BR. (2000). Natural selection, infectious transfer and the existence conditions for bacterial plasmids. Genetics 155: 1505-1519.

Bernhard K, Schrempf H, Goebel W. (1978). Bacteriocin and antibiotic resistance plasmids in Bacillus cereus and Bacillus subtilis. J Bacteriol 133: 897-903.

Bjorland J, Steinum T, Sunde M, Waage S, Heir E. (2003). Novel plasmid-borne gene qacJ mediates resistance to quaternary ammonium compounds in equine Staphylococcus aureus, Staphylococcus simulans, and Staphylococcus intermedius. Antimicrob Agents Chemother 47: 3046-3052.

Bouma JE, Lenski RE. (1988). Evolution of a bacteria/ plasmid association. Nature 335: 351-352.

Cao V, Lambert T, Courvalin P. (2002). ColE1-like plasmid pIP843 of Klebsiella pneumoniae encoding extendedspectrum beta-lactamase CTX-M-17. Antimicrob Agents Chemother 46: 1212-1217.

Chen CY, Lindsey RL, Strobaugh TP, Frye JG, Meinersmann RJ. (2010). Prevalence of ColE1-like plasmids and kanamycin resistance genes in Salmonella enterica serovars. Appl Environ Microbiol 76: 6707-6714.

Choi KH, Schweizer HP. (2006). mini-Tn7 insertion in bacteria with single attTn7 sites: example Pseudomonas aeruginosa. Nat Protoc 1: 153-161.

Clinical and Laboratory Standards Institute (2009). Performance Standards for Antimicrobial Susceptibility Testing Approved standard M100-S19, 19th edn. CLSI: Wayne, PA, USA.

Dahlberg C, Chao L. (2003). Amelioration of the cost of conjugative plasmid carriage in Eschericha coli K12. Genetics 165: 1641-1649.

Dionisio F, Conceição IC, Marques AC, Fernandes L, Gordo I. (2005). The evolution of a conjugative plasmid and its ability to increase bacterial fitness. Biol Lett 1: 250-252.

Doyle M, Fookes M, Ivens A, Mangan MW, Wain J, Dorman CJ. (2007). An H-NS-like stealth protein aids horizontal DNA transmission in bacteria. Science 315: 251-252.

Enne VI, Bennett PM, Livermore DM, Hall LM. (2004). Enhancement of host fitness by the sul2-coding plasmid p9123 in the absence of selective pressure. J Antimicrob Chemother 53: 958-963.

Freter R, Freter RR, Brickner H. (1983). Experimental and mathematical models of Escherichia coli plasmid transfer in vitro and in vivo. Infect Immun 39: 60-84.

Gogarten JP, Townsend JP. (2005). Horizontal gene transfer, genome innovation and evolution. Nat Rev Microbiol 3: 679-687.

Gordon DM. (1992). Rate of plasmid transfer among Escherichia coli strains isolated from natural populations. J Gen Microbiol 138: 17-21.

Granier SA, Hidalgo L, San Millan A, Escudero JA, Gutierrez B, Brisabois A et al. (2011). ArmA methyltransferase in a monophasic Salmonella enterica isolate from food. Antimicrob Agents Chemother 55: 5262-5266. 
Haines AS, Jones K, Batt SM, Kosheleva IA, Thomas CM. (2007). Sequence of plasmid pBS228 and reconstruction of the IncP-1alpha phylogeny. Plasmid 58: 76-83.

Haines AS, Jones K, Cheung M, Thomas CM. (2005). The IncP-6 plasmid Rms149 consists of a small mobilizable backbone with multiple large insertions. J Bacteriol 187: 4728-4738.

Hall AR, MacLean RC. (2011). Epistasis buffers the fitness effects of rifampicin- resistance mutations in Pseudomonas aeruginosa. Evolution 65: 2370-2379.

Harr B, Schlötterer C. (2006). Gene expression analysis indicates extensive genotype-specific crosstalk between the conjugative F-plasmid and the E. coli chromosome. BMC Microbiol 6: 80.

Harrison E, Brockhurst MA. (2012). Plasmid-mediated horizontal gene transfer is a coevolutionary process. Trends Microbiol 20: 262-267.

Harrison E, Koufopanou V, Burt A, Maclean RC. (2012). The cost of copy number in a selfish genetic element: the $2-\mu \mathrm{m}$ plasmid of Saccharomyces cerevisiae. J Evol Biol 25: 2348-2356.

Hauschild T, Lüthje P, Schwarz S. (2006). Characterization of a novel type of MLSB resistance plasmid from Staphylococcus saprophyticus carrying a constitutively expressed erm(C) gene. Vet Microbiol 115: 258-263.

Humphrey B, Thomson NR, Thomas CM, Brooks K, Sanders M, Delsol AA et al. (2012). Fitness of Escherichia coli strains carrying expressed and partially silent IncN and IncP1 plasmids. BMC Microbiol 12: 53 .

Itoh N, Kawanami T, Nitta C, Iwata N, Usami S, Abe Y et al. (2003). Characterization of pNI10 plasmid in Pseudomonas, and the construction of an improved Escherichia and Pseudomonas shuttle vector, pNUK73. Appl Microbiol Biotechnol 61: 240-246.

Itoh N, Koide Y, Fukuzawa H, Hirose S, Inukai T. (1991). Novel plasmid vectors for gene cloning in Pseudomonas. J Biochem 110: 614-621.

Kadlec K, Pomba CF, Couto N, Schwarz S. (2010). Small plasmids carrying $\operatorname{vga}(\mathrm{A})$ or $\operatorname{vga}(\mathrm{C})$ genes mediate resistance to lincosamides, pleuromutilins and streptogramin A antibiotics in methicillin-resistant Staphylococcus aureus ST398 from swine. J Antimicrob Chemother 65: 2692-2693.

Levin BR. (1993). The accessory genetic elements of bacteria: existence conditions and (co)evolution. Curr Opin Genet Dev 3: 849-854.

Lili LN, Britton NF, Feil EJ. (2007). The persistence of parasitic plasmids. Genetics 177: 399-405.

Martinez JL, Fajardo A, Garmendia L, Hernandez A, Linares JF, Martínez-Solano L et al. (2009). A global view of antibiotic resistance. FEMS Microbiol Rev 33: 44-65.

Mick V, Rebollo MJ, Lucía A, García MJ, Martín C, Aínsa JA. (2008). Transcriptional analysis of and resistance level conferred by the aminoglycoside acetyltransferase gene aac(2')-Id from Mycobacterium smegmatis. J Antimicrob Chemother 61: 39-45.

Modi RI, Castilla LH, Puskas-Rozsa S, Helling RB, Adams J. (1992). Genetic changes accompanying increased fitness in evolving populations of Escherichia coli. Genetics 130: 241-249.

Navarre WW, Porwollik S, Wang Y, McClelland M, Rosen $\mathrm{H}$, Libby SJ et al. (2006). Selective silencing of foreign DNA with low GC content by the H-NS protein in Salmonella. Science 313: 236-238.
Novick RP. (1987). Plasmid incompatibility. Microbiol Rev 51: 381-395.

Patnaik PR. (2000). An evaluation of models for the effect of plasmid copy number on bacterial growth rate. Biotechnol Lett 22: 1719-1725.

Paulsson J. (2002). Multileveled selection on plasmid replication. Genetics 161: 1373-1384.

Ponciano JM, De Gelder L, Top EM, Joyce P. (2007). The population biology of bacterial plasmids: a hidden Markov model approach. Genetics 176: 957-968.

Poulin R. (2013). Explaining variability in parasite aggregation levels among host samples. Parasitology 140: 541-546.

San Millan A, Escudero JA, Gutierrez B, Hidalgo L, Garcia N, Llagostera M et al. (2009). Multiresistance in Pasteurella multocida is mediated by coexistence of small plasmids. Antimicrob Agents Chemother 53: 3399-3404.

San Millan A, Garcia-Cobos S, Escudero JA, Hidalgo L, Gutierrez B, Carrilero L et al. (2010). Haemophilus influenzae clinical isolates with plasmid pB1000 bearing blaROB-1: fitness cost and interspecies dissemination. Antimicrob Agents Chemother 54: 1506-1511.

Sen D, Van der Auwera GA, Rogers LM, Thomas CM, Brown CJ, Top EM. (2011). Broad-host-range plasmids from agricultural soils have IncP-1 backbones with diverse accessory genes. Appl Environ Microbiol 77: 7975-7983.

Shaw DJ, Dobson AP. (1995). Patterns of macroparasite abundance and aggregation in wildlife populations: a quantitative review. Parasitology 111(Suppl): S111-S127.

Shintani M, Takahashi Y, Tokumaru H, Kadota K, Hara H, Miyakoshi M et al. (2010). Response of the Pseudomonas host chromosomal transcriptome to carriage of the IncP-7 plasmid pCAR1. Environ Microbiol 12: 1413-1426.

Silva RF, Mendonça SC, Carvalho LM, Reis AM, Gordo I, Trindade S et al. (2011). Pervasive sign epistasis between conjugative plasmids and drug-resistance chromosomal mutations. PLoS Genet 7: e1002181.

Simonsen L. (1991). The existence conditions for bacterial plasmids: theory and reality. Microb Ecol 22: 187-205.

Smets BF, Barkay T. (2005). Horizontal gene transfer: perspectives at a crossroads of scientific disciplines. Nat Rev Microbiol 3: 675-678.

Smillie C, Garcillán-Barcia MP, Francia MV, Rocha EP, de la Cruz F. (2010). Mobility of plasmids. Microbiol Mol Biol Rev 74: 434-452.

Stewart FM, Levin BR. (1977). The Population Biology of Bacterial Plasmids: A PRIORI Conditions for the Existence of Conjugationally Transmitted Factors. Genetics 87: 209-228.

Svara F, Rankin DJ. (2011). The evolution of plasmidcarried antibiotic resistance. BMC Evol Biol 11: 130.

Tato M, Coque TM, Baquero F, Cantón R. (2010). Dispersal of carbapenemase blaVIM-1 gene associated with different Tn402 variants, mercury transposons, and conjugative plasmids in Enterobacteriaceae and Pseudomonas aeruginosa. Antimicrob Agents Chemother 54: 320-327.

Toleman MA, Walsh TR. (2011). Combinatorial events of insertion sequences and ICE in Gram-negative bacteria. FEMS Microbiol Rev 35: 912-935. 
Trindade S, Sousa A, Xavier KB, Dionisio F, Ferreira MG, Gordo I. (2009). Positive epistasis drives the acquisition of multidrug resistance. PLoS Genet 5: e1000578.

Uhlin BE, Nordström K. (1977). R plasmid gene dosage effects in Escherichia coli K-12: copy mutants of the R plasmic R1drd-19. Plasmid 1: 1-7.

Verger JM, Grayon M, Chaslus-Dancla E, Meurisse M, Lafont JP. (1993). Conjugative transfer and in vitro/ in vivo stability of the broad-host-range IncP R751 plasmid in Brucella spp. Plasmid 29: 142-146.

Wang Y, Schwarz S, Shen Z, Zhang W, Qi J, Liu Y et al. (2012). Co-location of the multiresistance gene cfr and the novel streptomycin resistance gene aadY on a small plasmid in a porcine Bacillus strain. J Antimicrob Chemother 67: 1547-1549.

Wei Z, Yu T, Qi Y, Ji S, Shen P, Yu Y et al. (2011). Coexistence of plasmid-mediated KPC-2 and IMP-4 carbapenemases in isolates of Klebsiella pneumoniae from China. I Antimicrob Chemother 66: 2670-2671.

Yates CM, Shaw DJ, Roe AJ, Woolhouse ME, Amyes SG. (2006). Enhancement of bacterial competitive fitness by apramycin resistance plasmids from non-pathogenic Escherichia coli. Biol Lett 2: 463-465.

Yun CS, Suzuki C, Naito K, Takeda T, Takahashi Y, Sai F et al. (2010). Pmr, a histone-like protein H1 (H-NS) family protein encoded by the IncP-7 plasmid pCAR1, is a key global regulator that alters host function. J Bacteriol 192: 4720-4731.

Zar JH. (1974). Biostatistical Analysis. Prentice-Hall: Englewood Cliffs, NJ, USA.

Zioga A, Whichard JM, Kotsakis SD, Tzouvelekis LS, Tzelepi E, Miriagou V. (2009). CMY-31 and CMY-36 cephalosporinases encoded by ColE1-like plasmids. Antimicrob Agents Chemother 53: 1256-1259. (c) (1) (2) This work is licensed under a Creative ShareAlike 3.0 Unported License. To view a copy of this license, visit http://creativecommons.org/ licenses/by-nc-sa/3.0/

Supplementary Information accompanies this paper on The ISME Journal website (http://www.nature.com/ismej) 